ISSN: 1808-8759

\title{
CONSERVAÇÃO REFRIGERADA DE ABACATE 'HASS' E 'FUERTE' SUBMETIDOS À ATMOSFERAS MODIFICADAS ATIVAS
}

\author{
Viviane Citadini Russo ${ }^{1}$, Rogério Lopes Vieites ${ }^{2}$ \& Erica Regina Daiuto ${ }^{3}$
}

RESUMO: O objetivo deste trabalho foi avaliar a perda de massa e firmeza de frutos de abacate 'Hass' e 'Fuerte' submetidos à aplicação de atmosfera modificada ativa armazenados sob refrigeração. Os frutos foram selecionados, visando à homogeneização do lote quanto à ausência de injúrias e, em seguida foram lavados e higienizados. A lavagem foi feita com água e detergente e a higienização foi realizada com uma solução de hipoclorito de sódio a 1\%, por aproximadamente 20 minutos com o intuito de remover resíduos da colheita e micro-organismos aderidos à superfície antes da montagem do experimento. Os frutos das duas variedades foram acondicionados em embalagem de nylon+polietileno e submetidos a 5 tratamentos com diferentes mistura de gases, são eles: I-ambiente $(21,0 \mathrm{kPa}$ de O2+0,03 kPa de CO2); II - 4,0 kPa de O2+5,0kPa de CO2 ; III - 4,0 kPa de O2+6,0 kPa de CO2 ; IV - 4,0 kPa de $\mathrm{O} 2+7,0 \mathrm{kPa}$ de $\mathrm{CO} 2$ e $\mathrm{V}-4,0 \mathrm{kPa}$ de $\mathrm{O} 2+8,0 \mathrm{kPa}$ de $\mathrm{CO} 2$. Após os tratamentos, os frutos foram armazenados em câmara frigorífica a uma temperatura de $10^{\circ} \mathrm{C} \pm 1$ e umidade relativa de $90 \pm 5 \%$ e avaliados quanto a perda de massa e firmeza durante 35 dias, sendo as análises realizadas a cada 5 dias. Para abacates 'Hass', até os 25 dias de armazenamento os tratamentos IV e V, proporcionaram a menor perda de massa, aproximadamente $0,4 \%$. Para abacates 'Fuerte', o tratamento IV também resultou em menor percentual de perda de massa (aproximadamente 0,25\%) até o trigésimo dia de armazenamento. Não houve diferença significativa nos valores da firmeza para os frutos de abacate 'Hass', havendo interação entre os dias para os frutos de abacate 'Fuerte' que mostraram decréscimo significativos nos valores da firmeza já no $5^{\circ}$ dia de armazenamento mantendo-se constante até o final do período. Portanto, a atmosfera modificada associada ao armazenamento refrigerado foi efetiva na conservação dos abacates 'Hass' e 'Fuerte', resultando em baixo percentual de perda de massa e manutenção dos valores de firmeza.

PALAVRAS-CHAVE: Persea americana Mill., pós-colheita, embalagem.

\section{COLD STORAGE OF AVOCADO 'HASS' AND 'FUERTE' UNDERGOING ACTIVE MODIFIED ATMOSPHERES}

ABSTRACT: The objective of this work was to evaluate the weight loss and firmness of the different avocado types, 'Hass' and 'Fuerte', submitted to the application of active modified atmosphere and refrigerated storage. To get a homogenized lot, the fruits with no injuries were selected and then washed water and detergent in order to remove crop residues and microorganisms adhered to the surface. The avocado fruits were cleaned with sodium hypochlorite $1 \%$ solution for about 20 minutes before assembling the experiment. The two varieties of fruits were packed in polyethylene + nylon packing that were injected with mixture of gases constituting the treatments: I - the environment gas mixture (Iambiente (21,0 kPa de O2+0,03 kPa de CO2); II - 4,0 kPa de O2+5,0kPa de CO2 ; III - 4,0 kPa de O2+6,0 kPa de CO2 ; IV - 4,0 kPa de O2+7,0 kPa de CO2 e V - 4,0 kPa de O2+8,0 kPa de CO2. The fruits were stored in cold chamber at a temperature of $10 \pm 1^{\circ} \mathrm{C}$ and relative humidity of $90 \pm 5 \%$ and evaluated as the weigth loss and firmess for 35 days, with analyses performed every 5 days. For 'Hass' avocado until the 25th storage day, the treatments IV and V provided the lowest weight loss of approximately $0.4 \%$. For 'Fuerte" avocado, treatment IV also resulted in the lowest percentage of weight loss ( approximately $0.25 \%$ ) until the 30th storage day. There was no significant difference in the values of firmness between the fruits of avocado 'Hass', and the fruits of avocado'Fuerte' when considering the interection with days. The avocado 'Fuerte` showed a significant decreaseof firmness already on the 5th day of storage that remained constant until the end of the period.The modified atmosphere associated with the refrigerated storage was effective in the conservation of the 'Hass' and 'Fuerte' avocado fruits, resulting in low percentage of weight loss and maintenance of the firmness values.

KEYWORDS: Persea americana Mill, postharvest, packing.

\footnotetext{
${ }^{1}$ Aluna de mestrado na FCA/UNESP, Botucatu. E-mail: vivianecitadini@hotmail.com

${ }^{2}$ Professor titular na FCA/UNESP, Botucatu, e-mail: vieites@fca.unesp.br E-mail: vieites@fca.unesp.br

${ }^{3}$ E-mail: erdaiuto@yahoo.com.br
} 


\section{INTRODUÇÃO}

O abacate (Persea americana Mill.), visto no Brasil como fruta de "fundo de quintal", apresenta elevado valor nutritivo e é muito valorizado no mercado externo, sendo as variedades 'Hass' e 'Fuerte' as mais comercializadas. Estas duas variedades são diferenciadas por apresentarem menor diâmetro, maior teor de lipídios, casca mais grossa e menor teor de água, o que torna a polpa mais consistente possibilitando sua utilização em pratos salgados.

No Brasil a produção dos abacates 'Hass' e 'Fuerte' se concentra nos estados de São Paulo e Minas Gerais. No mercado brasileiro se encontram comercializados com a denominação de “avocado”, uma estratégia de marketing para valorizar o fruto e induzir o consumo do mesmo de maneira diferenciada, do mesmo modo que é utilizado nos outros países e não na forma de sobremesa como é de costume no Brasil. Grande parte da produção destes frutos destina-se a exportação e estes possuem selo de certificação Global Gap. O custo de produção do “avocado" é superior às variedades comercializadas no mercado brasileiro devido ao fato da colheita ser totalmente manual para atender o padrão de exportação.

O abacate é fruto climatérico cujo amadurecimento ocorre poucos dias após a colheita (HARDENBURG et al., 1986; SEYMOUR; TUCKER, 1993) e o comportamento pós-colheita pode ser influenciado pela temperatura e pelo tempo de armazenamento (TEIXEIRA et al., 1991). A temperatura adequada e o período para a conservação do abacate variam com a raça e a cultivar (ZAUBERMAN et al., 1973), pois estas apresentam sensibilidade diferente aos danos pelo frio. A literatura aponta estudos relacionados ao aumento do período de conservação de abacate, como avaliação da temperatura de armazenamento, uso de atmosfera modificada com aplicação de cera, irradiação gama, UVC e tratamento térmico (VIEITES et al., 2012).

O emprego da refrigeração prolonga o período de conservação dos frutos e o uso de atmosfera modificada durante o armazenamento pode reduzir os danos ocasionados pela respiração e pela transpiração, como perda e mudança da aparência (JERÔNIMO; KANESHIRO, 2000).

Visando evitar a perda de firmeza e, conseqüentemente prolongar a vida útil dos frutos, o presente trabalho avaliou o efeito de atmosferas modificada ativa na qualidade pós-colheita dos abacates 'Hass’ e ‘Fuerte’.

\section{MATERIAL E MÉTODOS}

Foram utilizados frutos de abacate 'Hass' e 'Fuerte', da safra de 2010, fornecidos pela empresa Jaguacy, localizada em Bauru/SP, cujas coordenadas geográficas são: latitude $22^{\circ} 19^{\prime} 18^{\prime \prime} \mathrm{S}$, longitude $49^{\circ} 04^{\prime} 13^{\prime \prime}$ W e $526 \mathrm{~m}$ de altitude, distante $90 \mathrm{~km}$ de Botucatu: latitude de $22^{\circ} 52^{\prime 2} 20^{\prime \prime}$ S, longitude $48^{\circ} 26^{\prime} 37^{\prime \prime} \mathrm{W}$ e $815 \mathrm{~m}$ de altitude. Os frutos depois de cuidadosamente colhidos no ponto de maturação fisiológica (de acordo com o teor de óleo, $21,6 \%$ ) foram imediatamente transportados para o Laboratório de Frutas e Hortaliças do Departamento de Gestão e Tecnologia Agroindustrial da Universidade Estadual Paulista “Júlio de Mesquita Filho" Faculdade de Ciências Agronômicas, Campus de Botucatu, SP. Os frutos foram inicialmente armazenados sob temperatura de $10^{\circ} \mathrm{C} \pm 1$, por 12 horas, para que ocorresse a diminuição do metabolismo dos frutos. Antes da instalação do experimento, os frutos foram selecionados visando à homogeneização do lote quanto à ausência de injúrias. Em seguida, os frutos foram lavados com água e detergente no intuito de remover resíduos da colheita e microrganismos aderidos à superfície, e higienizados com uma solução de hipoclorito de sódio a $1 \%$, por aproximadamente 20 minutos.

Após a seleção e higienização, os frutos de ambas as variedades foram submetidos a 4 tratamentos com atmosfera modificada ativa e acondicionados em embalagem de nylon + polietileno com dimensões de $30 \times 25 \times 0,14 c m$ e permeabilidade parcial à entrada e saída de $\mathrm{CO} 2$ e $\mathrm{O} 2$.

Nos tratamentos de II a V variou-se a concentração de $\mathrm{CO} 2$ e O2, sendo que o tratamento I é considerado como tratamento controle, ou seja, sem modificação da atmosfera interna da embalagem, conforme Tabela 1.

Tabela 1: Descrição dos tratamentos.

\begin{tabular}{ccc}
\hline Tratamentos & \% $\mathbf{O}_{2}$ & \% $\mathbf{C O}_{2}$ \\
\hline I & 21 & 0,03 \\
II & 4 & 5 \\
III & 4 & 6 \\
IV & 4 & 7 \\
V & 4 & 8 \\
\hline
\end{tabular}

A instalação do experimento consistiu no acondicionamento de três frutos de abacate por embalagem, sendo cada embalagem uma repetição, totalizando três embalagens por tratamento em cada dia de análise para cada variedade. As embalagens passaram pela injeção da mistura de gases de acordo com os tratamentos descritos na Tabela 1. O tratamento I (tratamento-controle, sem aplicação dos gases), foi apenas selado, constituindo-se da mistura de gases do próprio ambiente. 
Após a injeção de gases e selamento das embalagens, estas foram acondicionadas em câmara frigorífica a uma temperatura de $10^{\circ} \mathrm{C} \pm 1$ e umidade relativa de $90 \pm 5 \%$.

Nos abacates foram avaliados a perda de massa e firmeza dos frutos armazenados. Essas análises foram realizadas a cada 5 dias, sendo o total de 25 dias de análises para cada variedade.

A perda de massa foi obtida por pesagem dos frutos utilizando-se balança semi analítica da marca OWLABOR - carga máxima de $2000 \mathrm{~g}$ e precisão de 0,01g. As repetições foram pesadas no início do experimento e a cada 5 dias, permitindo o cálculo da perda de massa.

A firmeza foi obtida em texturômetro STEVENS LFRA Texture Analyser com uma distância de 20 mm e velocidade de $2,0 \mathrm{~mm} \mathrm{~s}-1$, utilizando-se o ponteiro TA 9/1000. A textura foi medida em dois pontos do mesmo fruto, e os resultados expressos em $\mathrm{Kgf} \mathrm{cm}-2$ que é definido como a força máxima requerida para que uma parte do ponteiro penetre na polpa do produto.

O experimento foi conduzido em delineamento inteiramente casualizado (DIC), compostos por cinco tratamentos e seis tempos de armazenamento, compondo um fatorial $5 \times 6$.

Para perda de massa, cada tratamento foi composto de três repetições, estas formadas por três sacos de nylon+polietileno para cada dia de análise. Para as avaliações de perda de massa fresca, foram utilizadas três repetições por tratamento ao longo do armazenamento.

Os resultados foram submetidos à análise de variância e as médias foram comparadas pelo teste Scott-Knott ao nível de $1 \%$ de probabilidade, conforme a característica avaliada.

\section{RESULTADOS E DISCUSSÃO}

\section{Perda de massa}

Para os frutos de abacate 'Hass' observou-se perda de massa fresca em todos os tratamentos ao longo dos 35 dias de armazenamento, sendo que o tratamento controle (T1) apresentou a maior perda de massa (Figura 1). Ocorreram oscilações das porcentagens de perda de massa fresca ao longo do período de armazenamento em todos os tratamentos por ocorrer acúmulo de água no interior de algumas embalagens. Este fato pode ser explicado pela transpiração dos frutos e devido a embalagem utilizada neste experimento possuir baixa permeabilidade ao vapor de água. O armazenamento refrigerado deve ter contribuído para que os valores de perda de massa fossem inferiores a 1,5\%. Até os 20 dias de armazenamento os tratamentos II, III e IV apresentaram a menor perda de massa. Após 35 dias de armazenamento, o tratamento II obteve o menor percentual de perda de massa em relação aos demais tratamentos.

O tratamento mais eficiente para a conservação da droga vegetal foi o sem vácuo refrigerado (SVR). Este tratamento proporcionou a melhor manutenção do teor de artemisinina durante o período de armazenamento, comparado aos demais tratamentos. Houve diferença significativa entre os tratamentos, sendo que aqueles mantidos sob-refrigeração foram os que melhor preservaram o principio ativo de A. annua. O fato das amostras terem sido armazenadas em geladeira, fez com que elas mantivessem o teor de água estáveis, ou até mesmo reduzido, fazendo com que a artemisinina sofresse uma degradação mais lenta durante todo o período. Além disso, não se verificou alterações significativas, em termos microbiológicos, entre os tratamentos aplicados.
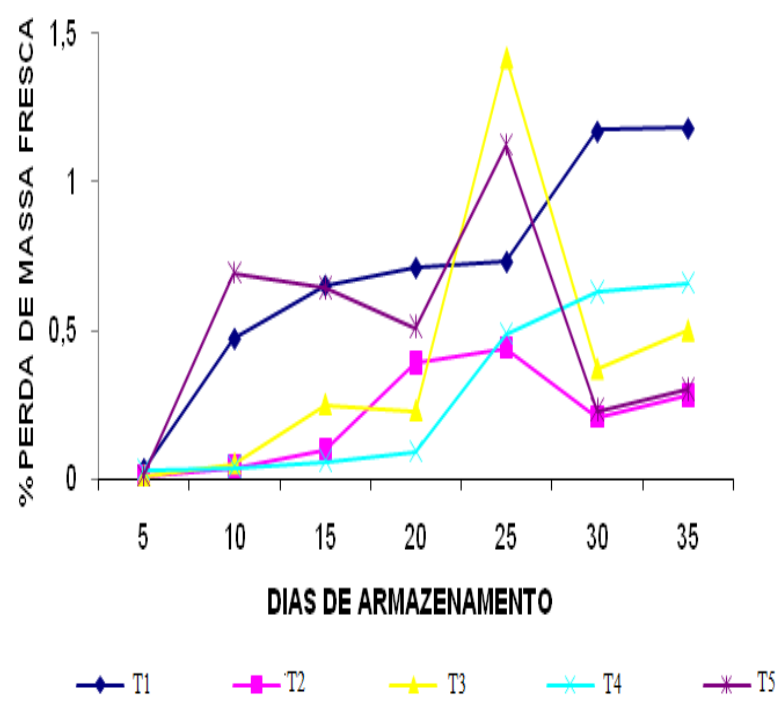

Legenda: $\mathrm{T} 1-21,0 \mathrm{kPa}$ de $\mathrm{O}_{2}+0,03 \mathrm{kPa}$ de $\mathrm{CO}_{2}$ ); T24,0 kPa de $\mathrm{O}_{2}+5,0 \mathrm{kPa}$ de $\mathrm{CO}_{2}$; T3 - 4,0 kPa de $\mathrm{O}_{2}+6,0$ $\mathrm{kPa}$ de $\mathrm{CO}_{2}$; T4 - 4,0 kPa de $\mathrm{O}_{2}+7,0 \mathrm{kPa}$ de $\mathrm{CO}_{2}$ e T5$4,0 \mathrm{kPa}$ de $\mathrm{O}_{2}+8,0 \mathrm{kPa}$ de $\mathrm{CO}_{2}$

Figura 1: Perda de massa (\%) em abacates 'Hass' submetidos à atmosfera modificada ativa armazenados em câmara frigorífica à $10 \pm 1^{\circ} \mathrm{C}$ com $90 \pm 5 \%$ de UR, por 35 dias. Legenda: T1- 0,03\% $\mathrm{CO}_{2} \mathrm{e}$ $21 \%$ de $\mathrm{O}_{2}$; T2- 5,0\% $\mathrm{CO}_{2}$ e $4,0 \% \mathrm{O}_{2}$; $\mathrm{T3}-6,0 \% \mathrm{CO}_{2}$ e $4,0 \% \mathrm{O}_{2} ; \mathrm{T} 4-7,0 \% \mathrm{CO}_{2}$ e $4,0 \% \mathrm{O}_{2} ; \mathrm{T} 5-8,0 \% \mathrm{CO}_{2}$ e $4,0 \% \mathrm{O}_{2}$.

Nos frutos de abacate 'Fuerte' observou-se perda de massa para todos os tratamentos ao longo do período de armazenamento. O tratamento IV mostrou perda de massa superior ao tratamento controle até o $25^{\circ}$ dia de armazenamento. No entanto, o percentual de perda de 
massa não foi superior a 1,5\% para os tratamentos, exceto para o tratamento III no $20^{\circ}$ dia de armazenamento. Deve-se ressaltar que, para a maioria dos produtos hortícolas frescos, a máxima perda de massa fresca tolerada para o não aparecimento de murcha e/ou enrugamento da superfície oscila entre 5 e 10\% (FINGER; VIEIRA, 2002) e produtos perecíveis como o abacate mesmo quando colocados em condições ideais, sofrem alguma perda de peso durante o armazenamento devido ao efeito combinado da respiraçã o e da transpiração (CHITARRA; CHITARRA, 2005). O tratamento II obteve a menor perda de massa quando comparada ao tratamento controle. As menores perdas de massa foram encontradas nos frutos dos tratamentos II e IV que, ao final do armazenamento, apresentaram perdas inferiores a 1,0\% (Figura 2).

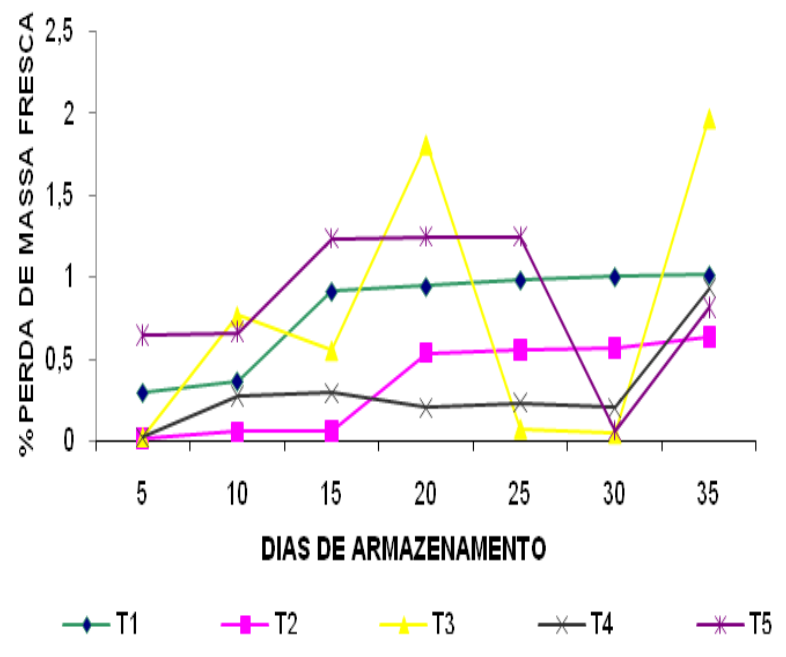

Legenda: $\mathrm{T} 1-21,0 \mathrm{kPa}$ de $\mathrm{O} 2+0,03 \mathrm{kPa}$ de $\mathrm{CO} 2)$; $\mathrm{T} 2-$ 4,0 kPa de $\mathrm{O} 2+5,0 \mathrm{kPa}$ de $\mathrm{CO} 2$; $\mathrm{T} 3-4,0 \mathrm{kPa}$ de $\mathrm{O} 2+6,0$ kPa de CO2 ; T4 - 4,0 kPa de O2+7,0 kPa de CO2 e T54,0 $\mathrm{kPa}$ de $\mathrm{O} 2+8,0 \mathrm{kPa}$ de $\mathrm{CO} 2$

Figura 2: Variação média da perda de massa fresca obtida em abacates 'Fuerte' submetidos à atmosfera modificada ativa armazenados em câmara frigorífica à $10 \pm 1^{\circ} \mathrm{C}$ com $90 \pm 5 \%$ de UR, por 35 dias.

Vieites et al. (2012) ao avaliar a qualidade pós colheita de abacates 'Fuerte' constataram que no armazenamento refrigerado a perda de massa não superou $2 \%$ do peso inicial dos frutos entretanto a perda de massa foi mais acentuada nos frutos mantidos sob temperatura ambiente. Daiuto et al. (2010) também observaram que nos frutos 'Hass' armazenados sob refrigeração, para qualquer tipo de tratamento físico realizado (irradiação gama, UV-C e tratamento térmico), a perda de massa foi inferior nos frutos armazenados sob temperatura ambiente. Os autores constataram também que a perda de massa foi mais acentuada após o $12^{\circ}$ dia de armazenamento dos frutos e ao avaliar a atividade respiratória dos frutos submetidos ao diferentes tratamentos físicos encontraram o pico no $9^{\circ}$ dia de armazenamento.

Os percentuais de perda de massa para qualquer um dos tratamentos físicos realizados por Daiuto et al. (2010), dos frutos mantidos sob refrigeração, foram superiores ao encontrados nesta pesquisa. Portanto a atmosfera modificada ativa, ou mesmo o tratamento controle deste experimento, contribuiu de forma efetiva para redução da perda de massa dos frutos das duas variedades, embora estas apresentassem comportamento diferente. Vale ressaltar que a concentração de $\mathrm{CO} 2$ do tratamento $\mathrm{V}$ não foi benéfica na conservação dos frutos, para as duas variedades em estudo.

Os abacates 'Hass' e 'Furte' apresentaram comportamento distinto para perda de massa, possivelmente devido comportamento fisiológico em função das variedades.

\section{Firmeza}

A perda de firmeza é normal durante a pós-colheita (BÁEZ-SAÑUDO et al., 2001), contudo, deve-se levar em consideração que a firmeza é um importante atributo na qualidade dos frutos, já que afeta a resistência ao transporte, ao ataque de microrganismos e a própria característica sensorial dos frutos.

Nesta pesquisa não houve diferença significativa nos valores da firmeza para os frutos de abacate 'Hass', havendo interação entre os dias para os frutos de abacate 'Fuerte' que mostraram decréscimo significativos nos valores da firmeza já no $5^{\circ}$ dia de armazenamento mantendo-se constante até o final do período quando analisada a média geral de dias (Tabela 2). 
Tabela 2: Firmeza ( $\mathrm{gf} \mathrm{cm}-2$ ) obtida em abacates 'Hass' e 'Fuerte' submetidos à atmosfera modificada ativa armazenados em câmara frigorífica a $10 \pm 1^{\circ} \mathrm{C}$ com $90 \pm 5 \%$ de UR, por 25 dias.

\begin{tabular}{|c|c|c|c|c|c|c|c|}
\hline \multicolumn{8}{|c|}{ 'Hass' } \\
\hline \multirow{2}{*}{$\begin{array}{l}\text { Concen- } \\
\text { trações }\end{array}$} & \multicolumn{6}{|c|}{ Dias de armazenamento } & \multirow{2}{*}{$\begin{array}{l}\text { Média geral } \\
\text { de } \\
\text { concentraçõ } \\
\mathbf{s}\end{array}$} \\
\hline & $\mathbf{0}$ & 5 & 10 & 15 & 20 & 25 & \\
\hline $\mathbf{I}$ & 1018,5 & 1000,5 & 956,3 & 1018,9 & 1015,1 & 1017,7 & $1004,5 \pm 24,61$ \\
\hline II & 1018,5 & 1004,8 & 1021,2 & 987,0 & 1019,1 & 993,8 & $1007,4 \pm 14,55$ \\
\hline III & 1018,5 & 1013,9 & 1022,4 & 1019,5 & 1017,2 & 1022,5 & $1019,0 \pm 3,27$ \\
\hline IV & 1018,5 & 1015,7 & 1021,1 & 1028,8 & 1015,0 & 965,0 & $1010,7 \pm 22,93$ \\
\hline $\mathbf{v}$ & 1018,5 & 1009,4 & 1014,3 & 879,3 & 1018,3 & 1014,4 & $992,4 \pm 55,50$ \\
\hline $\begin{array}{l}\begin{array}{l}\text { Média } \\
\text { geral de } \\
\text { dias }\end{array} \\
\end{array}$ & 1018,5 a & $\begin{array}{c}1008,9 \mathrm{a} \\
\pm 6,30\end{array}$ & $\begin{array}{c}1007,0 \mathrm{a} \\
\pm 28,55\end{array}$ & $\begin{array}{l}986,7 \text { a } \\
\pm 62,09\end{array}$ & $\begin{array}{c}1016,9 \text { a } \\
\pm 1,85\end{array}$ & $\begin{array}{c}1002,7 \mathrm{a} \\
\pm 23,74\end{array}$ & \\
\hline
\end{tabular}

'Fuerte'

\begin{tabular}{|c|c|c|c|c|c|c|c|}
\hline \multirow{2}{*}{$\begin{array}{l}\text { Concen- } \\
\text { trações }\end{array}$} & \multicolumn{6}{|c|}{ Dias de armazenamento } & \multirow{2}{*}{$\begin{array}{c}\text { Média geral } \\
\text { de } \\
\text { concentraçõe } \\
\text { s }\end{array}$} \\
\hline & $\mathbf{0}$ & 5 & 10 & 15 & 20 & 25 & \\
\hline I & 1038,5 & 959,5 & 913,5 & 919,4 & 988,8 & 1019,4 & $931,7 \pm 80,53$ a \\
\hline II & 1038,5 & 973,5 & 923,4 & 975,2 & 1011,5 & 1011,3 & $947,4 \pm 83,85$ a \\
\hline III & 1038,5 & 956,0 & 973,0 & 935,1 & 965,0 & 1000,6 & $936,5 \pm 75,16$ a \\
\hline IV & 1038,5 & 979,7 & 884,4 & 914,4 & 1010,8 & 1009,7 & $931,4 \pm 86,49$ a \\
\hline v & 1038,5 & 903,2 & 1012,0 & 1015,1 & 1005,3 & 985,0 & $951,7 \pm 89,77$ a \\
\hline $\begin{array}{l}\text { Média } \\
\text { geral de } \\
\text { dias }\end{array}$ & $1038,5 \mathrm{a}$ & $\begin{array}{l}954,4 \mathrm{~b} \\
\pm 30,23\end{array}$ & $\begin{array}{c}941,3 \mathrm{~b} \\
\pm 50,82\end{array}$ & $\begin{array}{l}951,8 \mathrm{~b} \\
\pm 42,67\end{array}$ & $\begin{array}{c}956,2 \mathrm{~b} \\
\pm 19,73\end{array}$ & $\begin{array}{c}1005,2 \mathrm{~b} \\
\pm 13,12\end{array}$ & \\
\hline $\begin{array}{l}\text { Médias } \\
\text { diferem } \\
\text { probabil }\end{array}$ & $\begin{array}{l}\text { segu } \\
\text { entr }\end{array}$ & $\begin{array}{l}\text { as } \\
\text { si }\end{array}$ & $\begin{array}{l}\text { mes } \\
\text { lo } \mathrm{T}\end{array}$ & $\begin{array}{l}\text { let } \\
\text { te } \mathrm{S}\end{array}$ & $\mathrm{pa}$ & $\begin{array}{c}\text { os } \mathrm{d} \\
\mathrm{tt} \text { à }\end{array}$ & $\begin{array}{l}\text { ias não } \\
1 \% \text { de }\end{array}$ \\
\hline
\end{tabular}

Legenda: T1- 21,0 kPa de O2+0,03 kPa de CO2); T24,0 kPa de $\mathrm{O} 2+5,0 \mathrm{kPa}$ de $\mathrm{CO} 2$; $\mathrm{T} 3-4,0 \mathrm{kPa}$ de $\mathrm{O} 2+6,0$ $\mathrm{kPa}$ de $\mathrm{CO} 2$; $\mathrm{T} 4$ - 4,0 kPa de O2+7,0 kPa de CO2 e T54,0 kPa de $\mathrm{O} 2+8,0 \mathrm{kPa}$ de $\mathrm{CO} 2$

Segundo Chitarra e Chitarra (2005), este decréscimo se deve ao fato da firmeza estar estreitamente relacionada com a solubilização de substâncias pécticas e que durante a maturação ocorre a conversão da pectina insolúvel em pectina solúvel, amolecendo e diminuindo a resistência dos frutos.

Vieites et al. (2012) observaram que a melhor preservação da firmeza dos abacates 'Fuerte' foi observada quando os frutos foram refrigerados, enquanto para os frutos armazenados em temperatura ambiente o decréscimo foi gradual e constante, para a temperatura de refrigeração, observou-se aumento nos valores de firmeza e queda apenas após o pico respiratório ( $\left.9^{\circ} \mathrm{dia}\right)$.

Oliveira et al. (2000) ao avaliar frutos de abacate 'Fuerte' submetidos aplicação de cera vegetal não encontraram diferença entre tratamentos, que diferiam pelo tipo de cera utilizada e concentração, entretanto, em relação aos dias de armazenamento, os autores observaram que os valores de firmeza decresceram.

Ben-Arie e Zutkhi (1992) verificaram que o uso da atmosfera modificada ativa em caqui também retarda a perda de firmeza e inibe o desenvolvimento de desordens na polpa e epiderme dos frutos. Neste experimento, o uso da atmosfera modificada juntamente com o armazenamento refrigerado nos frutos de abacate 'Hass' e 'Fuerte' apresentou o mesmo efeito.

\section{CONCLUSÃO}

A atmosfera modificada associada ao armazenamento refrigerado foi efetiva na conservação dos abacates 'Hass' e 'Fuerte', resultando em baixo percentual de perda de massa e manutenção dos valores de firmeza. No entanto, para as duas variedades dos frutos submetidos à pressão parcial de 4,0 kPa de O2+7,0 kPa de CO2.

\section{AGRADECIMENTOS}

À Coordenação de Aperfeiçoamento de Pessoal de Nível Superior (CAPES) e à empresa Jaguacy pelo fornecimento dos frutos.

\section{REFERÊNCIAS}

BÁEZ-SAÑUDO, R. et al. Evaluación de películas comestibles sobre la vida postcosecha del mango.

Proceedings. of the International. Society. Tropical Horticultural, Miami, v. 41, p. 172-178, 2001.

BEN-ARIE, R.; ZUTKHI, Y. Extending the storage life of 'Fuyu' persimmon by modified-atmosphere packaging. Hortscience, Alexandria, v. 27, n. 7, p. 811813, 1992.

CHITARRA, M. I. F.; CHITARRA, A. B. Pós-colheita de frutos e hortaliças: fisiologia e manuseio. Lavras: Ed UFLA, 2005. 785 p.

DAIUTO, E. R. et al. Taxa respiratória de abacate 'hass' submetido a diferentes tratamentos físicos. Revista Iberoamericana de Tecnología Postcosecha, México, v. 10, n. 2, p. 101-109, 2010.

FINGER, F. L.; VIEIRA, G. Controle da perda póscolheita de água em produtos hortícolas. Viçosa: UFV, 2002. $29 \mathrm{p}$.

JERÔNIMO, E. M.; KANESHIRO, M. A. B. Efeito da associação de armazenamento sob refrigeração e atmosfera modificada na qualidade de mangas 'Palmer'. Revista Brasileira de Fruticultura, Jaboticabal, v. 22, n. 2, p. 237-243, 2000. 
HARDENBURG, R. E.; WATADA. A. E.; WANG, C. $Y$. The comercial storage of fruits, vegetables, and florist and nursery stocks. Beltsville: USDA, 1986. 130 p.

OLIVEIRA, M. A. et al. Ceras para conservação pós colheita de frutos de abacateiro fuerte, armazenados em temperatura ambiente. Scientia Agricola, Piracicaba, v. 57, n. 4, p. 777-780, 2000.

SEYMOUR, G. B.; TUCKER, G. A. Avocado. In: SEYMOUR, G. B.; TAYLOR, J. E.; TUCKER, G. A. Biochemistry of fruit ripening. London: Chapman \& Hall, 1993. p. 53-76.

TEIXEIRA, C. G. et al. Abacate: cultura, matériaprima, processamento e aspectos econômicos. Campinas: ITAL, 1991. $250 \mathrm{p}$.

VIEITES, R. L.; DAIUTO, E. R.; FUMES. J. F. F. Capacidade antioxidante e qualidade pós colheita de abacate 'Fuerte'. Revista Brasileira de Fruticultura, Jaboticabal, v. 34, n. 2, p. 336-348, 2012.

ZAUBERMAN, M. S.; SCHIFFMAN-NADEL, M.; YANKO, U. Susceptibility to chilling injury of three avocado cultivars stages of ripening. HortScience, Alexandria, v. 8, n. 4, p. 511-513, 1973. 